\title{
Effet de l'incorporation de matières grasses dans les concentrés sur la croissance et la qualité des carcasses chez l'agneau mâle de bergerie, sevré précocement durant l'hiver
}

\author{
G Bozzolo 1, M Bouillier-Oudot ${ }^{1}$ \\ avec la collaboration technique de M Rizqi ${ }^{1}$, D Grasset ${ }^{2}, \mathrm{H}_{\text {Manse }}{ }^{1}$ \\ 1 ENSAT, 145, avenue de Muret, 31076 Toulouse cedex; \\ 2 GEBRO, centre d'élevage de la Pommière, Lauras, 12250 Roquefort, France
}

(Reçu le 13 septembre 1993; accepté le 15 mai 1994)

\begin{abstract}
Résumé - Les effets de 2 régimes expérimentaux ont été comparés à ceux d'une alimentation classique sur la croissance et la qualité des carcasses d'agneaux à partir de 270 mâles Lacaune, répartis en 3 lots : i) utilisation de $21 \mathrm{~kg}$ de starter commercial puis d'un aliment de finition commercial ; ii) utilisation de $21 \mathrm{~kg}$ de starter enrichi en huile de coprah (5\%) puis d'un aliment de finition enrichi en saindoux $(3 \%)$; iii) utilisation de $17 \mathrm{~kg}$ de starter enrichi en coprah puis d'un aliment de finition commercial. Les animaux furent contrôlés individuellement et abattus à poids constant $(39 \mathrm{~kg})$. Durant la période de démarrage, l'huile de coprah n'a pas apporté de modification sur le rythme de croissance. En revanche, celle-ci, associée au régime saindoux (traitement ii), a induit un effet différé en période de finition, améliorant l'indice de consommation $(3,6$ vs $3,0 \mathrm{~kg} \mathrm{MS} / \mathrm{kg}$ de gain ; $P<0,05)$. La prolongation de la distribution de l'aliment starter a permis une croissance en finition légèrement supérieure $(+5 \%$, $P<0,04)$. L'indice de consommation des 3 lots s'est détérioré progressivement jusqu'à un seuil correspondant à environ $31 \%$ du poids de maturité. La croissance relative a atteint un maximum $(17,5 \mathrm{~g} / \mathrm{j} / \mathrm{kg}$ Pvif) à un stade correspondant à $22 \%$ du poids de maturité, puis a diminué régulièrement jusqu'au terme (10 g/j/kg de Pvif). Concernant les caractéristiques de carcasse, seule l'huile de coprah introduite au démarrage a permis de réduire la proportion de carcasses au tissu gras externe mou et huileux $(17 \%$ vs $33 \%$ ). Aucune amélioration n'est apparue concernant la couleur du tissu gras de couverture.
\end{abstract}

agneau / coprah / saindoux / croissance / carcasse

Summary - Effect of fat supply to fattening diet on growth and carcass qualities of early weaned male lambs fed indoors during winter. The effect of dietary fat supplies was investigated 
for growth and carcass characteristics on 270 Lacaune male-lambs randomized and fattened in 3 pens indoors during winter. Two treatments were compared with classical starter and fattening diets: i) $21 \mathrm{~kg}$ commercial starter then a commercial 'growing-fattening' diet; ii) $21 \mathrm{~kg}$ starter supplemented with coconut oil then the 'growing-fattening' diet supplemented with lard; iii) $17 \mathrm{~kg}$ starter supplemented with coconut oil then the reference commercial 'growing-fattening' diet. The 2 starting diets were isocaloric and isonitrogenous ( $M E=10.9 \mathrm{MJ} / \mathrm{kg} \mathrm{DM} ; C P=189 \mathrm{~g} / \mathrm{kg} \mathrm{DM}$ ). One was supplemented with $5 \%$ coconut oil (fat $(F)=74 \mathrm{~g} / \mathrm{kg} \mathrm{DM}$ ) and fed at 2 levels $(21 \mathrm{~kg} / \mathrm{lamb}$ during $29 \mathrm{dvs} 17 \mathrm{~kg} / \mathrm{lamb}$ for $21 \mathrm{~d})$. The second was a reference commercial starter diet $(F=31.6 \mathrm{~g} / \mathrm{kg} \mathrm{DM})$ issued at $21 \mathrm{~kg} / \mathrm{lamb}$ level. The 2 succeeding growing-finishing diets were also both isocaloric and isonitrogenous ( $M E=10.7 \mathrm{MJ} / \mathrm{kg}$ $D M ; C P=176 \mathrm{~g} / \mathrm{kg} D M)$. One was supplemented with $3 \%$ lard $(F=50.4 \mathrm{~g} / \mathrm{kg} \mathrm{DM})$ and the other was a reference commercial diet $(F=37.5 \mathrm{~g} / \mathrm{kg} D M$ ). All diets were given ad libitum and straw was freely accessible. The lambs were weaned early (3-5 weeks old) and were slaughtered at a fixed body weight $(B W=39 \mathrm{~kg})$ in one slaughterhouse. Carcasses were scored by the same skilled operator. From these, and for statistical purposes owing the discrepancy in the frequency distribution of initial parameters of lambs, 2 sub-samples were made and standardized according to a normal distribution and in respect to the weight at weaning $(12 \mathrm{~kg} ; 3 \times[\mathrm{n}=58])$, the relative growth between $0-20 \mathrm{~d}(16.2$ $g / d / g B W ; 3 \times[n=64])$. Concerning growth, feeding with starter diet over an extended time produced a higher growth rate on final fattening stage $(+5 \%, \mathrm{P}<0.04)$. Coconut oil did not promote growth during the starting stage. However, through a remaining effect, it improved the conversion food ratio in the last fattening stage when lard diet was associated (CFR $=3.6$ for treatment a vs $3.0 \mathrm{~kg} \mathrm{DM} / \mathrm{kg}$ gain; $\mathrm{P}<0.05$ ). Supplementing with lard in the 'growing-finishing' diet did not modify the lamb growth. In the 3 lots, the conversion food ratio increased until the lambs reached about $32 \%$ of their mature weight. The relative growth came close to a maximum when lambs weighed about $22 \%$ of $B W$ at maturity (17.5 g/kg BW), then it regularly decreased to $10 \mathrm{~g} / \mathrm{kg} \mathrm{BW}$ at the end. Concerning the characteristics of carcasses, only coconut oil supplementation during the starting stage induced a greater proportion of carcasses with very firm subcutaneous fat $(27 \mathrm{vs} 13 \%, \mathrm{P}<0.03)$ and reduced the proportion of soft and oily class of disqualitative carcasses (17vs $33 \%, \mathrm{P}<0.02$ ). However the colours of the layer fat were not affected.

lamb / coconut oil / lard / growth / carcass

\section{INTRODUCTION}

Chez les ruminants, l'utilisation des matières grasses dans les concentrés pour renforcer l'apport énergétique, ou pour orienter la qualité des productions, est d'actualité. C'est notamment le cas pour les vaches à haut niveau de production laitière en raison de leur forte demande énergétique. Chez les ruminants destinés à produire de la viande l'incorporation de lipides alimentaires est peu fréquente. En effet, leur emploi conduit à un surcoût de la formulation et leur incidence est souvent négative sur le niveau d'ingestion au-delà d'un taux de $10 \%$ (Christie, 1981 ; Bauchart et al, 1985 ; Bock et al, 1991). Par ailleurs, chez l'agneau, le risque de produire des carcasses trop grasses, élément déterminant de leur valorisation, constitue un handicap majeur. Cependant, pour certaines catégories d'agneaux élevés en bergerie, avec des régimes riches en concentrés, une dépréciation commerciale vient s'ajouter lorsque le tissu gras externe des carcasses manque de fermeté et/ou que sa couleur s'éloigne de la teinte blanche. La fréquence de ces défauts peut être particulièrement élevée parmi les agneaux issus des troupeaux laitiers, traditionnellement sevrés tôt. Chez les agneaux Lacaune engraissés en bergerie avec des concentrés dès l'âge de 20 à $40 \mathrm{j}$, plus de $30 \%$ sont affectés (Bozzolo et al, 1992). Dans ces conditions particulières, le rôle des matières grasses peut intervenir à plusieurs stades : 
- Durant la période de transition de postsevrage, caractérisée par un déficit énergétique important (stress, adaptation au nouveau régime alimentaire), l'introduction de lipides riches en acides gras de moyennes et courtes chaînes carbonées peut constituer une alternative intéressante. Compte tenu de leur forte digestibilité et de leur rapide oxydation hépatique, ceux-ci fournissent un carburant énergétique efficace (Frost et Wells, 1981 ; Cera et al, 1989). Ce remplacement d'une partie de l'amidon de la ration par des matières grasses peut aussi réduire les conséquences indésirables d'une orientation propionique trop prononcée (acides gras insaturés, ramifiés et impairs) génératrice de graisses molles (Garton et al, 1972 ; Ørskov et al, 1975). Les précédents essais que nous avons réalisés sur agneaux mâles (Bozzolo et al, 1991, 1993a) ont confirmé ce point de vue.

- Pendant la phase d'engraissement-finition, lorsque l'accrétion des lipides dans les dépôts s'intensifie (Christie, 1981 ; Aurousseau, 1986), l'emploi de lipides riches en acides gras longs peut conduire à des dépôts fermes (Devier et Pfander, 1974 ; Bock et al, 1991). En effet les acides gras insaturés d'origine alimentaire subissent une biohydrogénation dans le rumen, avant d'être incorporés majoritairement dans les triglycérides adipocytaires (Christie, 1981 ; Annison, 1984). Par ailleurs, l'activité des désaturases adipocytaires, ordinairement importante chez les Ovins et associée à la synthèse de novo (Wahle, 1974 ; St John et al, 1991), paraît ralentie en présence d'acides gras exogènes (Wahle, 1974 ; Chang et al, 1992). Les essais que nous avons conduits en introduisant du saindoux à un faible niveau d'incorporation $(30 \mathrm{~g} / \mathrm{kg}$ de $\mathrm{MB}$ ) dans l'aliment concentré offert à des agneaux mâles et femelles (Bozzolo et al, 1993b) ont indiqué une tendance à produire des carcasses plus fermes et plus blanches, sans pour autant détériorer les performances de croissance.
II restait à considérer la combinaison successive de ces 2 apports au travers des aliments de démarrage et d'engraissementfinition, ce qui constitue l'objet principal de ce travail. Par ailleurs, I'hypothèse d'une possible interférence de la quantité d'aliment de démarrage consommée avant la transition vers l'aliment de finition, fait également l'objet de cette investigation.

\section{MATÉRIEL ET MÉTHODES}

\section{Animaux}

Cet essai a porté sur un ensemble de 270 agneaux mâles de race Lacaune-lait répartis en 3 lots logés dans des cases contiguës (centre d'engraissement du GEBRO) de janvier à mai 1992. Au départ, la répartition des agneaux sevrés précocement (entre 3 et 5 sem) dans les différentes cases a été conduite en considérant leur poids au sevrage $\left(P_{0}\right.$ compris entre 9 et $17 \mathrm{~kg}$ ) dispersé selon une loi normale autour d'une moyenne de $13 \mathrm{~kg}$ et par classe de $2 \mathrm{~kg}$.

Les animaux ont été nourris à volonté à l'aide d'aliments concentrés granulés, complémentés par de la paille mise à libre disposition dans des râteliers. Leur abattage a été effectué sur la base d'un poids constant, dès lors que les agneaux franchissaient le poids de $39 \mathrm{~kg}$. Celui-ci était contrôlé régulièrement par une pesée hebdomadaire.

\section{Régimes alimentaires}

Plusieurs combinaisons d'aliments et de niveaux dans la distribution, portant à la fois sur l'aliment de "démarrage» et sur l'aliment "d'engraissement-finition" (quantité consommée et qualité), ont été exploitées. Chacun des aliments a été formulé sur une base isoénergétique et isoazotée en faisant varier les proportions des matières premières et par incorporation de matières grasses (tableau I).

Les 3 lots d'agneaux $(3 \times[n=90)$ ont été conduits de la manière suivante : 
Tableau I. Composition des aliments d'engraissement.

\section{Aliments de démarrage \\ $D t$ (témoin) $\quad D c$ (coprah)}

Aliments d'engraissement-finition Ft (témoin) Fs (saindoux)

1.a. Composition en matières premières

Matières premières $(\mathrm{g} / \mathrm{kg}$ de matière brute)

$\begin{array}{lcc}\text { Céréales } & 420 & 280 \\ \text { Drèches } & 50 & 16,6 \\ \text { Pulpe d'agrumes } & 50 & 50,8 \\ \text { Son } & 12,5 & - \\ \text { Luzerne déshydratée } & 100 & 200 \\ \text { Pois } & 100 & 100 \\ \text { Tourteaux de soja } & 143,7 & 118,2 \\ \text { Tourteaux de tournesol } & 11,3 & 80 \\ \text { Mélasse de canne } & 50 & 50 \\ \text { Graisse d'os (Bovosol) } & 6 & - \\ \text { Huile de coprah } & - & 50 \\ \text { Saindoux } & - & - \\ \text { Minéraux } & 43,5 & 41,4 \\ \text { Adjuvant vitaminique Z15 } & 3 & 3 \\ \text { Additif Z74 (avotan) } & - & - \\ \text { Additif Z88 (lasalocide) } & 4 & 4 \\ \text { Additif Z20 (oxytétracycline) } & 5 & 5\end{array}$

I.b. Composition chimique et valeur énergétique

Constituants ( $/ \mathrm{kg} \mathrm{MS}$ )

Matière sèche (MS)

Matière organique

898,6

897,0

882,3

887,5

Lignine

142,3

114,5

37,7

28,9

188,3

31,6

74,0

Matières grasses

0,96

0,97

11,11
885,4

908,0

116,8

134,3

$\begin{array}{cc}377,5 & 282,8 \\ 125 & 125 \\ 120 & 98,3 \\ 23,3 & 12,8 \\ 74,4 & 120 \\ 100 & 150 \\ 78 & 30,3 \\ - & 61,8 \\ 50 & 50 \\ 8 & - \\ - & - \\ - & 30 \\ 41,8 & 37 \\ - & - \\ 2 & 2 \\ - & - \\ - & -\end{array}$

31,4

36,7

180,6

50,4

$0,93 \quad 0,95$

$10,62 \quad 10,87$

I.c. Composition en acides gras les plus représentatifs de la matière grasse Acides gras $(\mathrm{g} / 100 \mathrm{~g} \text { des esters méthyliques totaux })^{\mathrm{b}}$

$\begin{array}{lrrrr}\text { C12 } & 0,1 & 27,0 & 2,5 & 0,2 \\ \text { C14 } & 0,7 & 10,2 & 1,7 & 1,2 \\ \text { C16 } & 18,1 & 13,0 & 17,5 & 21,1 \\ \text { C18 } & 5,7 & 4,1 & 6,2 & 9,0 \\ \text { C18: } 1 & 23,0 & 14,7 & 24,1 & 30,1 \\ \text { C18:2 } & 44,4 & 23,4 & 36,4 & 31,1 \\ \text { C18: } & 5,2 & 2,9 & 5,0 & 3,7 \\ \text { EAG saturés } & 25,8 & 58,2 & 31,7 & 32,4 \\ \text { EAG insaturés } & 74,0 & 41,8 & 68,3 & 67,6 \\ \text { IAG < C15 } & 1,2 & 40,5 & 7,8 & 1,9\end{array}$

a UFV, EM : estimations à partir des équations de prédiction en fonction de la composition chimique des aliments selon Giger-Reverdin et al (1990). b Détermination par chromatographie en phase gazeuse après extraction des matières grasses selon la méthode de Folch et al (1957) et préparation des esters méthyliques par transméthylation (AFNOR, NFT 60-223, 1977). 
- le lot $\mathrm{Dt}_{(21)} \mathrm{Ft}$, servant de référence, a reçu l'aliment de démarrage témoin à raison de $21 \mathrm{~kg}$ en moyenne par agneau soit durant $29 \mathrm{j}+5 \mathrm{j}$ de transition, puis l'aliment de finition témoin ;

- le lot $\mathrm{Dc}_{(21)} \mathrm{Fs}$ combinait l'incorporation d'huile de coprah dans l'aliment de démarrage et celle de saindoux dans l'aliment de finition. L'aliment $\mathrm{Dc}_{(21)}$ a été distribué selon les mêmes conditions que précédemment ;

- le lot $\mathrm{Dc}_{(17)} \mathrm{Ft}$ a été nourri avec l'aliment de démarrage "coprah" à raison de $17 \mathrm{~kg}$ par agneau, soit 21 j plus 5 j de transition afin de vérifier la probable influence de la quantité de concentré distribué au démarrage sur la croissance des agneaux et sur la qualité des carcasses.

Les 2 aliments de démarrage (D : 0,97 UFV/kg MS ; $189 \mathrm{~g}$ de MAT/kg MS) se distinguaient par la présence d'huile de coprah à raison de $50 \mathrm{~g} / \mathrm{kg}$ $\mathrm{MB}$ dans l'un (Dc) comparée à $6 \mathrm{~g} / \mathrm{kg} \mathrm{MB}$ de Bovosol (graisse d'os) dans l'aliment commercial témoin $(D t)$.

Après une transition de $5 \mathrm{j}$, les aliments «d'engraissement-finition» ( $F$ : UFV $=0,94 / \mathrm{kg} M S$; MAT $=175 \mathrm{~g} / \mathrm{kg} \mathrm{MS}$ ) ont été substitués aux précédents et distribués à volonté jusqu'au terme de l'essai. Les 2 aliments comparés différaient par la présence de $30 \mathrm{~g} / \mathrm{kg}$ MB de saindoux dans l'un (Fs) et de $8 \mathrm{~g}$ de Bovosol dans l'autre ( $\mathrm{Ft}$ ), aliment commercial utilisé comme témoin.

\section{Paramètres observés}

\section{Paramètres d'élevage}

Les agneaux ont été pesés individuellement tous les $20 \mathrm{j}$ jusqu'à $60 \mathrm{j}\left(\mathrm{P}_{0}, \mathrm{P}_{20}, \mathrm{P}_{40}, \mathrm{P}_{60}\right)$, puis hebdomadairement jusqu'à l'abattage $\left(P_{A b}\right)$. Les vitesses de croissance journalières correspondantes (GMQ) ont été calculées pour apprécier les différentes séquences de croissance liées à la distribution des 2 aliments successifs. L'âge en début d'engraissement $\left(\mathrm{Ag}_{0}\right)$, la modalité de naissance (NAIS : simple, double ou triple), ainsi que la durée d'engraissement (DUEN) ont été notés.

Un indice de consommation moyen (IC) a été calculé pour chacune des périodes correspondantes, et une estimation de l'écart type moyen a été approchée selon les indications rapportées par Bozzolo et al (1992).

\section{Paramètres.de carcasse}

La totalité des agneaux a été abattue dans le même abattoir. Les observations sur carcasse ont été réalisées par le même opérateur, après ressuage en chambre froide. Cette opération a été conduite selon une procédure accélérée en maintenant les carcasses, après stimulation électrique et abattage, en atmosphère ventilée à la température de $-20^{\circ} \mathrm{C}$ pendant $2 \mathrm{~h}$.

Les différents critères suivants ont été relevés : le poids de carcasse à froid (PCAR), le rendement de carcasse dérivé (RDT $=\mathrm{PCAR} /\left(\mathrm{P}_{\mathrm{Ab}} \mathrm{X}\right.$ $0,95)$ ), la notation de la conformation (NCONF) et celle du développement du gras de couverture (NGEXT) selon la classification EUROPA. L'épaisseur de gras dorsal (EPGDO) a été mesurée en $\mathrm{mm}$ à partir d'une fente pratiquée au scalpel à hauteur et latéralement aux $1^{\mathrm{re}}$ et $2^{\mathrm{e}}$ vertèbres lombaires. L'importance des dépôts de graisse intercostale (NGIC) selon une échelle de 9 classes comprises entre 1 et 5 et celle de gras périrénal (NGRO en g) sont aussi rapportées. La qualité du tissu gras sous-cutané a été appréciée par une note de fermeté (NTEN) en 4 classes définies par Bouillier-Oudot et al (1992) (NTEN 1 = gras mou et huileux; NTEN 4 = gras très ferme) et une note de couleur en 7 classes par référence au nuancier Munsell (NCOUL : $1=$ blanc, 2 = blanc crème, 3 = rosée, $4=$ bistre clair, $5=$ brun rouge, $6=$ jaune clair, $7=$ bistre clair avec reflets verdâtres).

\section{Traitement des résultats}

Compte tenu des mortalités intervenues en cours d'engraissement, des pertes d'identification (carcasses) et de quelques agneaux saisis (au total $12,11,10$ agneaux respectivement pour les lots $\mathrm{Dt}_{(21)} \mathrm{Ft}, \mathrm{Dc}_{(21)} \mathrm{Fs}$ et $\mathrm{Dc}_{(17)} \mathrm{Ft}$ ), les comparaisons statistiques ont été réalisées sur 2 échantillonnages standardisés sur la base d'options présentant les lots sous des angles d'analyse différents. Le principe retenu a été de réunir le plus grand nombre d'agneaux aussi semblables que possible dans les différents lots, en fonction de critères initiaux, et selon une distribution conforme à la loi normale (fig 1). En effet, la présence d'une interaction significative entre périodes de mesures et régimes, révélée par une analyse préalable de la variance généralisée (test de Wilks : $P<$ 0,003 ), ne permettait pas de procéder à un traitement par l'analyse de la covariance. 
Le premier échantillonnage (1) a été construit à partir du poids au sevrage $\left(P_{0}\right)$. Cet indicateur, en effet, peut être considéré comme un critère synthétique des particularités anté-sevrage des agneaux (poids à la naissance, origine génétique, mode de naissance, importance de l'allaitement maternel, âge au sevrage). Les individus retenus possédaient un poids compris entre 9 et $15 \mathrm{~kg}$, la moyenne étant de $12 \mathrm{~kg}$ et les effectifs restant équilibrés dans chaque classe de $1 \mathrm{~kg}$

\section{Echantillon 1}
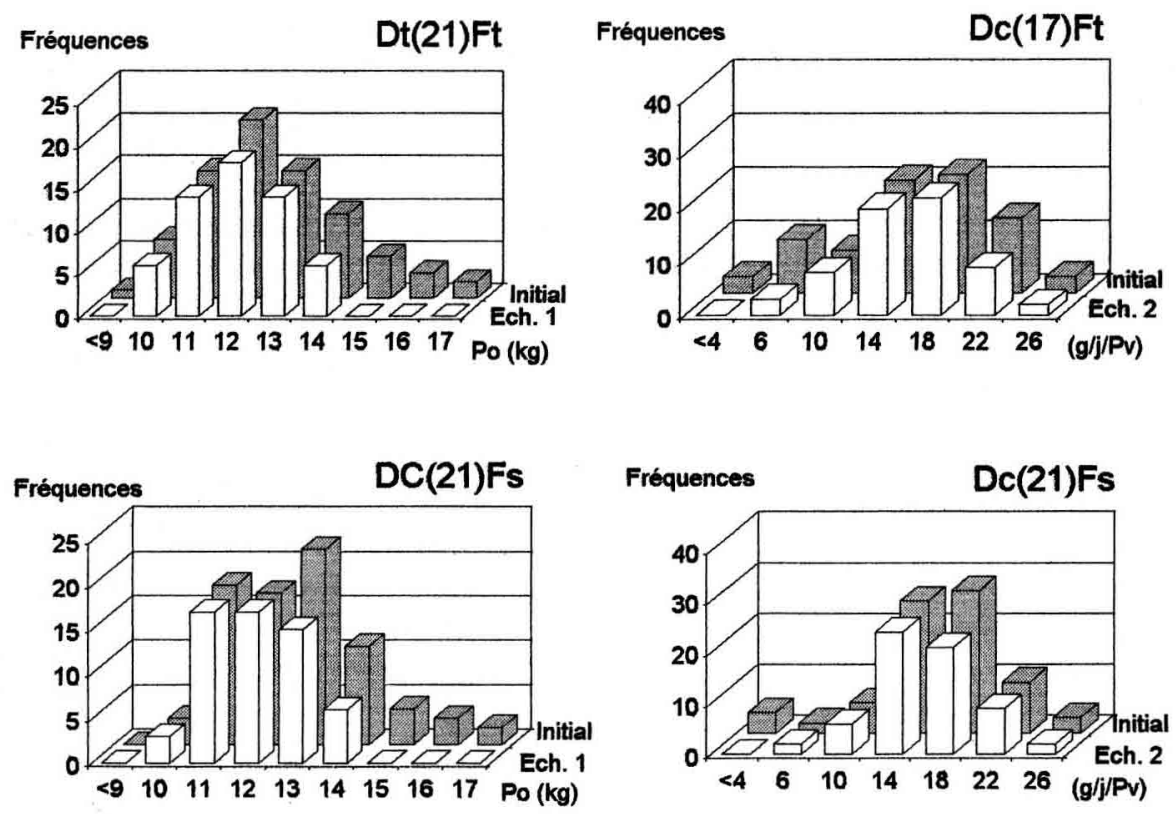
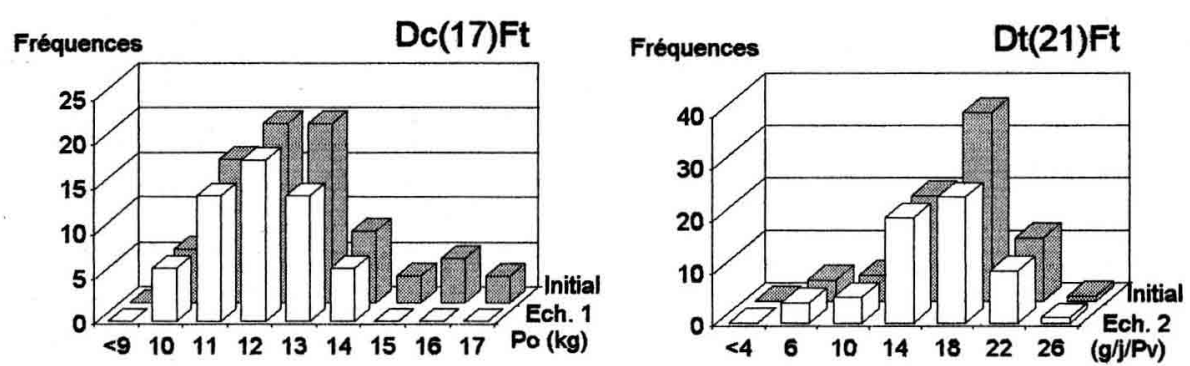

Fig 1. Standardisation des échantillons - Histogrammes des effectifs. Initial : répartition des fréquences d'agneaux présents au moment de l'abattage dans les différents lots : $\mathrm{Dt}_{(21)} \mathrm{Ft}(n=78)$, $\mathrm{Dc}_{(21)} \mathrm{Fs}(n=79)$ et $\mathrm{Dc}_{(17)} \mathrm{Ft}(n=80)$. Éch 1 : échantillonnage en fonction du poids en début d'engraissement ( $P 0=12 \mathrm{~kg} ; \mathrm{Sd}=1,1 \mathrm{~kg} ; n=58)$. Éch 2 : échantillonnage en fonction de la croissance relative entre 0 et $20 \mathrm{j}$ d'engraissement $\left(\mathrm{CR}_{0-20 \mathrm{j}}=16,2 \mathrm{gj}^{-1} \mathrm{kgPv}^{-1} ; \mathrm{Sd}=4,3 \mathrm{~g} ; n=64\right)$. $\mathrm{Dt}_{(21)} \mathrm{Ft}$ : aliment de démarrage témoin $(21 \mathrm{~kg})$ + aliment de finition témoin ; $\mathrm{Dc}{ }_{(21)} \mathrm{Fs}$ : aliment de démarrage $(21 \mathrm{~kg})$ supplémenté en huile de coprah $(5 \%)+$ aliment de finition supplémenté en saindoux $(3 \%)$. $\mathrm{Dc}_{(17)} \mathrm{Ft}$ : aliment de démarrage coprah $(17 \mathrm{~kg})+$ aliment de finition témoin. 
d'écart. Au total 58 agneaux par lot ont pu satisfaire à ces contraintes.

Le second échantillonnage (2) a eu pour double objectif d'homogénéiser les animaux en fonction de leur niveau d'adaptation après sevrage, et de comparer les effets induits par les régimes alimentaires postérieurement à cette transition, notamment pour le lot $\mathrm{Dc}_{(17)} \mathrm{Ft}$. En effet, la croissance relative réalisée entre 0 et 20 j d'engraissement peut être considérée comme le reflet indirect du comportement d'adaptation (stress de post-sevrage, comportement dans le groupe, résistance aux maladies, adaptation digestive, variabilité génétique). Cet échantillon a été bâti selon le même principe que précédemment en retenant les agneaux couvrant la plage de $\mathrm{CR}_{0-20 j}$ comprise entre 4 et $28 \mathrm{gj}^{-1} \mathrm{Pv}^{-1}$ et centrée sur une moyenne de $16 \mathrm{~g}$. Les effectifs d'agneaux ont été équilibrés par classe de $4 \mathrm{~g}$. Au total 64 individus par lot ont satisfait à ces conditions.

Les résultats bruts de l'essai sont fournis à titre comparatif. Indices de consommation et croissances relatives sont également confrontés à partir de l'ensemble des agneaux présents dans les lots. Les différentes variables de nature quantitative des 3 échantillons sont analysées par la décomposition de la variance et les moyennes sont comparées par le test de Newman Keuls à $P<0,05$ lorsque le $F$ calculé est significatif à $P<0,05$. Les comparaisons des variables de nature qualitative et/ou ordinale sont abordées à l'aide du test $\chi^{2}$.

\section{RÉSULTATS ET DISCUSSION}

\section{Croissance et efficacité alimentaire}

Pour l'ensemble des 3 échantillons analysés (tableau II), l'âge en début d'engraissement et le type de naissance ne diffèrent pas significativement et n'expliquent pas les quelques différences observées.

\section{Échantillon 1}

Lorsque les agneaux sont standardisés sur le poids au sevrage, les vitesses de croissance enregistrées entre 0 et $20 \mathrm{j}$, pendant l'adaptation de post-sevrage, ne sont pas significativement différentes en fonction de la nature des aliments de démarrage. Si l'on considère les $\mathrm{GMQ}_{20-40 \mathrm{j}}$ et $\mathrm{GMQ}_{40-60 \mathrm{j}}$, la différence s'accentue entre les lots soumis aux régimes $\mathrm{Dc}_{(17)}$ et $\mathrm{D}_{(21)}$. Le premier croît plus lentement $(-4,8 \%, P<0,20 ;-10 \%$, $P<0,01$ respectivement), de sorte que sur l'ensemble de l'engraissement $\left(G M Q_{0-A b}\right)$, par cumul, la croissance est inférieure à celle réalisée par le régime $\mathrm{Dc}_{(21)} \mathrm{Ft}(-5 \%, P$ $<0,03)$. La durée d'engraissement est en conséquence plus longue que pour le lot témoin (90 j vs 84 j pour $\left.\mathrm{Dc}_{(17)} \mathrm{Ft} ; P<0,04\right)$, $\mathrm{Dc}_{(21)} \mathrm{Fs}$ étant situé en position intermédiaire.

Ces résultats infirment en apparence l'amélioration que nous avions enregistrée précédemment, liée à l'incorporation d'huile de coprah ( $+63 \%$ sur la période $0-20 \mathrm{j}$; Bozzolo et al, 1991, 1993a). Plusieurs explications possibles peuvent être à l'origine de ce constat. Les agneaux dont nous disposions pour cette expérience étaient globalement plus lourds à l'entrée $\left(\mathrm{P}_{0}=13 \mathrm{vs}\right.$ $12 \mathrm{~kg}$ et les poids intra lots moins variables : CV $=9 \%$ vs $12 \%$ les années passées). Par ailleurs, les températures de I'hiver 1992 furent plus clémentes dans la période considérée, induisant certainement une demande énergétique moindre. Enfin, la densité d'agneaux dans les cases d'engraissement (90 agneaux/case contre 120/case dans les essais précédents) a pu diminuer la concurrence entre animaux et l'intensité du stress. L'ensemble de ces éléments s'est probablement additionné pour réduire le déficit énergétique des agneaux pendant la transition post-sevrage. D'ailleurs, nous avions précédemment observé que cet effet était surtout marqué sur des agneaux légers au moment du sevrage $\left(P_{o}<11 \mathrm{~kg}\right)$.

$\mathrm{Si}$ on observe les vitesses de croissance réalisées avec les aliments de finition témoin et l'aliment enrichi en saindoux $\left(\mathrm{Dt}_{(21)} \mathrm{Ft}\right.$ vs $\left.\mathrm{Dc}_{(21)} \mathrm{Fs}\right)$ les performances sont peu différentes, ce qui rejoint les conclusions des 


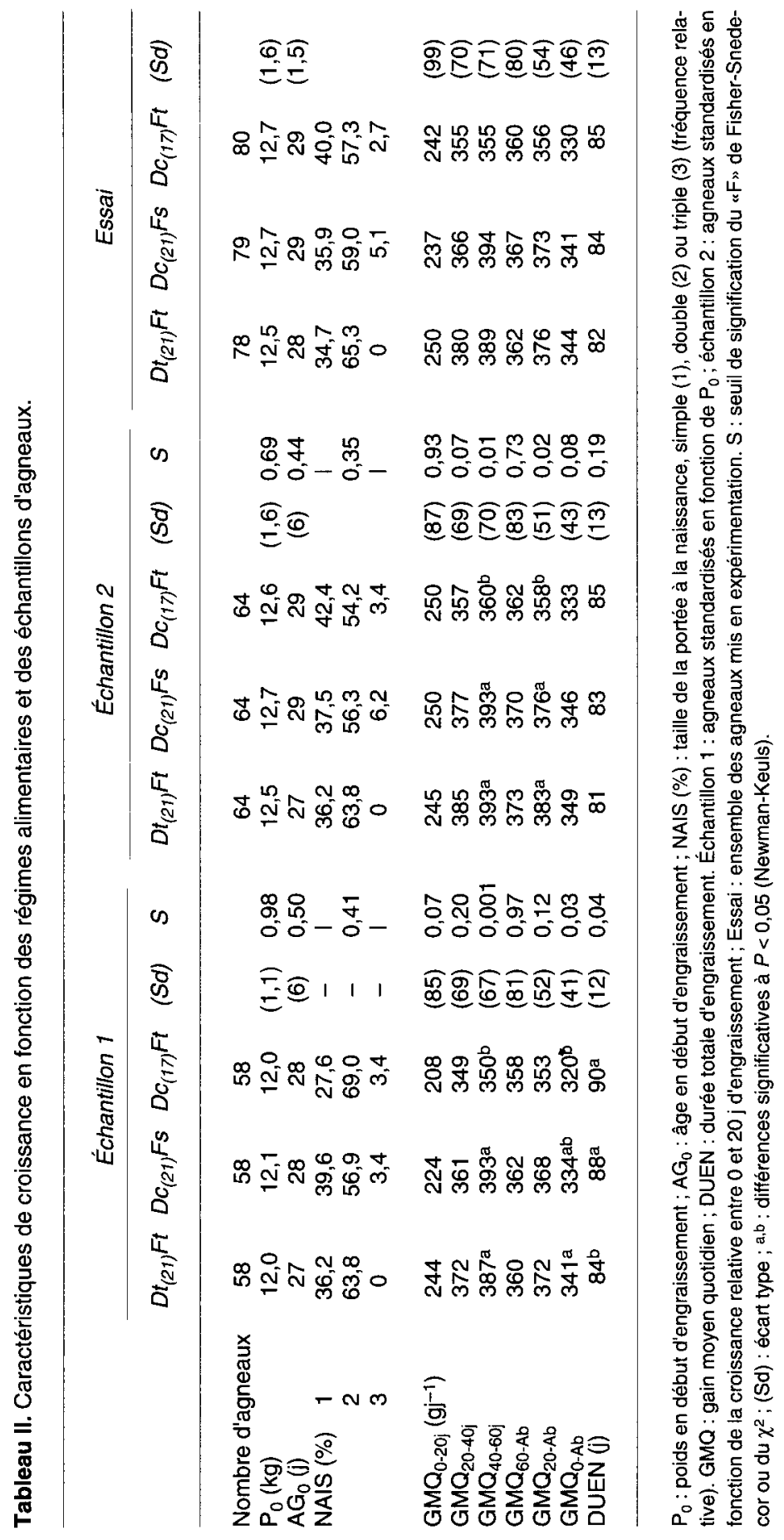


essais précédents. Cependant, la distribution en moindre quantité de l'aliment de démarrage (17 kg vs $21 \mathrm{~kg}$ ) semble provoquer une croissance plus faible en finition.

\section{Échantillon 2}

Dès lors que les agneaux sont standardisés par rapport à leur croissance durant la phase d'adaptation $\left(\mathrm{CR}_{0-20 \mathrm{j}}=16 \mathrm{gj}^{-1} \mathrm{Pv}^{-1}\right.$; $\mathrm{CV}=27 \%$ ), les différences apparaissent plus nettement, en fonction de la quantité d'aliment de démarrage reçue, dès le changement d'aliment $(P<0,07)$. À partir d'un $\mathrm{GMQ}_{0-20 j}$ identique, au-delà, les différences s'accentuent notamment entre 40 et $60 \mathrm{j}$ au détriment du lot $\mathrm{Dc}_{(17)} \mathrm{Ft}\left(\mathrm{GMQ}_{40-60 \mathrm{j}}:-8,4 \%\right.$, $P<0,01)$, conduisant à une différence significative sur la période de finition $\left(\mathrm{GMQ}_{20-\mathrm{Ab}}\right.$ $=-5,7 \%, P<0,02)$. Au total, sur l'ensemble de l'engraissement $\left(\mathrm{GMQ}_{0-\mathrm{Ab}}\right)$, la croissance est légèrement plus faible pour le lot $\mathrm{Dc}_{(17)} \mathrm{Ft}$ ( $333 \mathrm{~g}$ vs $348 \mathrm{~g}$ en moyenne pour les 2 autres lots, contraste significatif à $P<0,05$ ). Ceci confirme que, lorsque les disparités au démarrage sont corrigées, l'effet de la nature des aliments d'engraissement-finition est peu différent. Seule, la durée consacrée à la distribution de l'aliment de démar- rage paraît influente. II est probable que la différence de $5 \mathrm{~kg}$ d'aliment en moyenne/agneau, soit $8 \mathrm{j}$ dans la distribution de l'aliment de démarrage, induise un meilleur approvisionnement pour cette phase d'anabolisme protéique intense (Andrews et Ørskov, 1970 ; Thériez et al, 1981), compte tenu des plus fortes concentrations énergétique et azotée des aliments starter, mais aussi de la présence d'adjuvants spécifiques (complexe vitaminique, lasalocide et oxytétracycline).

L'analyse de l'évolution des indices de consommation selon les périodes d'engraissement ainsi que celle des croissances relatives (fig 2) montre bien une identité de comportement entre lot témoin et lots complémentés à l'huile de coprah durant la période $0-20 \mathrm{j}$. Entre $20 \mathrm{j}$ d'engraissement et $60 \mathrm{j}$, les évolutions ne sont pas significativement différentes. Après $60 \mathrm{j}$, les agneaux complémentés avec de l'huile de coprah au démarrage paraissent mieux utiliser l'aliment de finition, notamment lorsqu'il est enrichi en saindoux (IC = 3,0 vs 3,6 pour le lot témoin, $P<0,05)$. La position intermédiaire pour le lot $\mathrm{Dc}_{(17)} \mathrm{Ft}(\mathrm{IC}=3,27)$ peut laisser supposer que l'huile de coprah provoque un effet rémanent puisqu'elle tend à

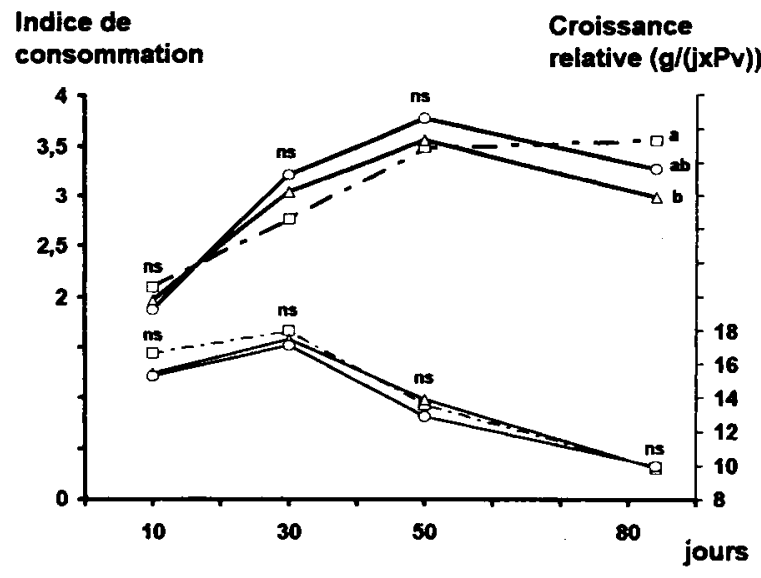

Fig 2. Évolution des indices de consommation et de la croissance relative en fonction du temps d'engraissement et selon le régime d'engraissement : -- $\square--\mathrm{Dt}_{(21)} \mathrm{Ft} ;-\Delta-$ $\mathrm{Dc}_{(21)} \mathrm{Fs} ;-\mathrm{O}-\mathrm{Dc}_{(17)} \mathrm{Ft} ;-$ indice de consommation $(\mathrm{kg}$ de $\mathrm{MS} / \mathrm{kg}$ de gain) ; -- croissance relative $\left(\mathrm{gi}^{-1} \mathrm{kgPv}^{-1}\right) . \mathrm{a}, \mathrm{b}, \mathrm{c}$ différences significatives à $P<0,05$, ns (non significatif) ; test de Newman-Keuls. 
améliorer l'efficacité alimentaire du régime de finition témoin $(\mathrm{Ft})$ alors que la croissance relative est semblable pour l'ensemble des lots à ce même stade (CR = $\left.10,0 \mathrm{gj}^{-1} \mathrm{Pv}^{-1}, P>0,94\right)$. Ceci est à relier à un conditionnement du métabolisme, antérieur au stade de $30 \%$ du poids de maturité, déjà soulevé par Aurousseau et al (1973). En effet, l'évolution générale de l'indice de consommation montre sa détérioration jusqu'à un seuil situé entre 40 et $60 \mathrm{j}$ d'engraissement $\left(\mathrm{IC}_{0-20 \mathrm{j}}=1,98 ; \mathrm{IC}_{40-60 \mathrm{j}}=\right.$ $3,61)$ confirmant nos résultats antérieurs sur les 2 sexes. Cet événement marque certainement les effets conjugués de l'adaptation digestive au régime de finition (fonctionnalité du rumen) à ce stade charnière ( $P 50 \mathrm{j}=29,2 \mathrm{~kg}$ en moyenne pour les 3 lots, $P>0,32$ ) et la modification de la composition du croît corporel si l'on se reporte à la dégradation progressive et linéaire de la croissance relative, pour les 3 lots, dès le stade antérieur $\left(\mathrm{CR}_{20-40 \mathrm{j}}: 17,5 \mathrm{~g} / \mathrm{l}\right)$ jusqu'au stade terminal $\left(\mathrm{CR}_{60-\mathrm{Ab}}=10,0 \mathrm{~g} / \mathrm{j}\right)$. Ce point correspond à un poids moyen équivalent à $22 \%$ du poids de maturité (fig 2).

Au plan de la croissance, l'huile de coprah ne paraît donc présenter d'intérêt que lorsque les conditions d'environnement sont sévères et que les agneaux sont légers au sevrage $(<11 \mathrm{~kg})$ grâce à l'oxydation rapide de ses acides gras à chaîne carbonée de faible et moyenne longueur (Frost et Wells, 1981). Pour des agneaux de poids moyen $(12 \mathrm{~kg}$ ) ou lourds (> $14 \mathrm{~kg}$ ), l'avantage est minime ou même absent. La composition pondérale des lots (poids individuel des agneaux au sevrage) ressort donc comme un facteur déterminant et peut expliquer les résultats contradictoires souvent cités à ce sujet, notamment chez le porcelet (Benevenga et al, 1989 ; Cera et al, 1989 ; Mahan, 1991). En revanche, l'effet rémanent de l'huile de coprah en cours de finition (indice de consommation), stade normalement lié à l'adipogenèse (Turgeon et al, 1986), semble s'inscrire dans une orien- tation métabolique particulière et peut donc interférer avec la qualité des tissus adipeux.

\section{Qualité des carcasses}

Parmi les paramètres de carcasses (tableau III), au travers des 2 différents échantillonnages, les seuls éléments qui présentent une tendance constante à la différence sont le poids carcasse et la notation de la fermeté. Le premier (PCAR) est supérieur pour le lot ayant bénéficié des 2 apports de matières grasses. Cependant, l'aspect significatif de cette faible différence est à mettre au compte de la variabilité intra lot réduite de ce critère en raison du protocole d'abattage à poids fixé. De plus, ces carcasses présentent en moyenne une conformation légèrement meilleure.

\section{Fermeté du tissu gras}

La note de fermeté (NTEN) est, en moyenne, systématiquement plus élevée pour les lots ayant bénéficié de la supplémentation en huile de coprah dans l'aliment starter $(+0,5$ point $(P<0,05) ;+0,4$ point $(P<0,03)$, pour les échantillons 1 et 2$)$. L'analyse plus détaillée de la répartition des fréquences (tableau III) montre effectivement que cette différence porte plus spécifiquement sur les classes extrêmes de fermeté (NTEN 1 : 16,8\% vs 32,8\% pour le lot témoin $(P<0,02)$, et NTEN $4: 27,1 \%$ vs $13,2 \%(P<0,03)$ en moyenne sur les 2 échantillons). En revanche, les proportions de carcasses classées en qualité intermédiaire ne diffèrent pas, soit en moyenne : $27,5 \%$ et $27,8 \%$ respectivement pour NTEN 2 et NTEN 3.

Les caractéristiques d'engraissement (épaisseur du gras dorsal et poids du gras périrénal) sont significativement supérieures pour le lot $\mathrm{Dc}_{(21)}$ Fs dans l'échantillon 1, mais elles restent d'assez faible amplitude $(+0,5$ 


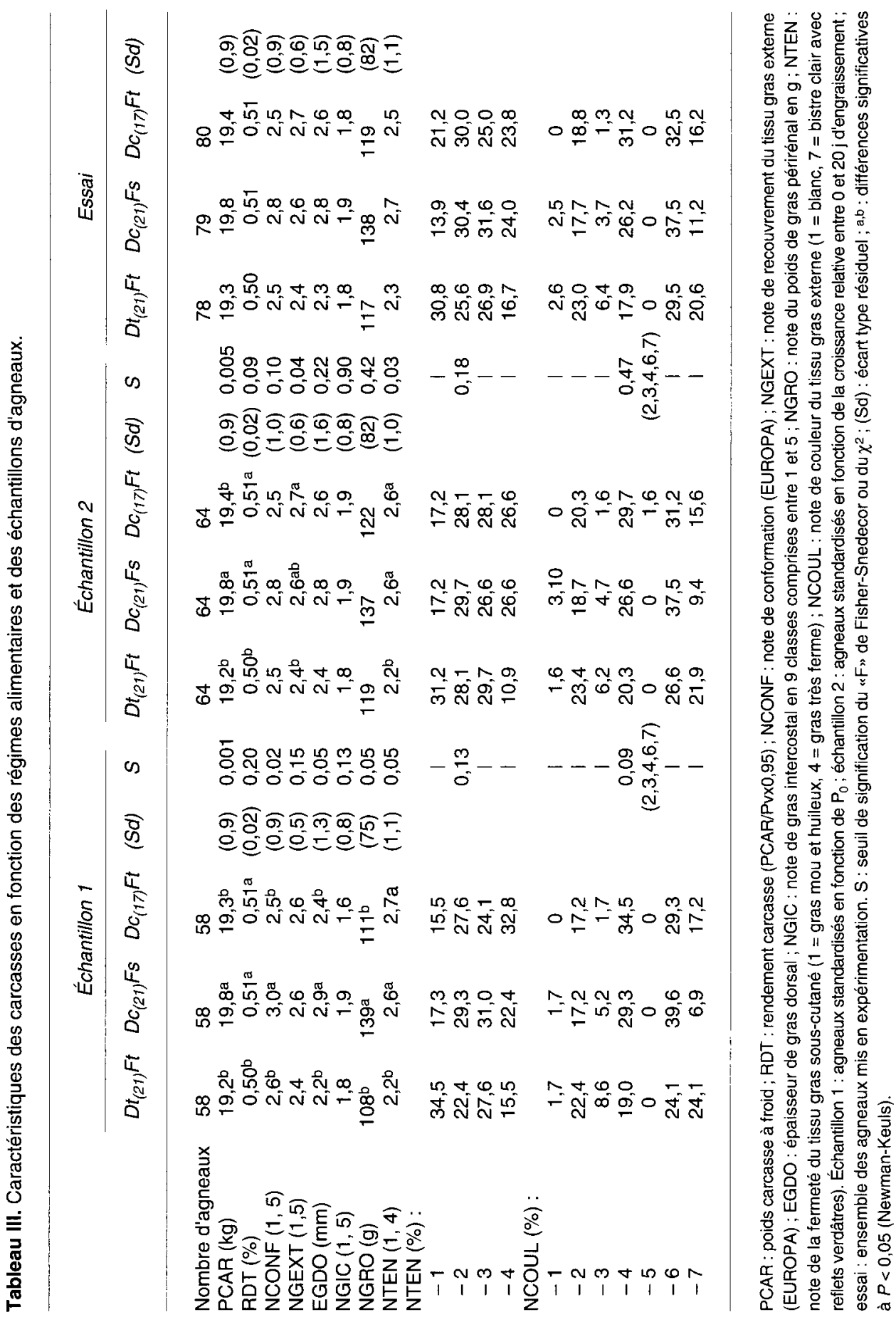


point). En revanche, seule une légère tendance apparaît lorsque les agneaux sont équilibrés par rapport à leur comportement en post-sevrage (Ech 2).

En conséquence, il ressort que dans cet essai seule l'influence de l'huile de coprah, quel que soit le rythme de distribution de l'aliment starter (17 kg ou $21 \mathrm{~kg}$ ), a produit une légère amélioration de la fermeté des gras de couverture en modifiant les proportions dans les classes extrêmes. L'addition de saindoux ne paraît pas avoir apporté de bonification, les lots $\mathrm{Dc}_{(21)}$ Fs et $\mathrm{DC}_{(17)} \mathrm{Ft}$ présentant des résultats semblables.

Ces résultats confirment partiellement ceux précédemment obtenus (Bozzolo et al, 1991, 1992b) avec les agneaux les plus lourds et soumis à un régime complémenté en huile de coprah. Aurousseau et al (1973) émettaient l'hypothèse d'un conditionnement du métabolisme des adipocytes par les constituants de l'huile de coprah, conduisant à la présence d'acides gras plus saturés dans les tissus adipeux. Ce point de vue pourrait être retenu compte tenu de la rémanence observée. En effet, dans cet essai, l'effet du coprah n'est pas marqué sur la croissance des agneaux durant la période initiale de transition. En outre, à ce stade, l'adipogenèse est encore peu active (Bénévent, 1971 ; Andrew et Ørskov, 1970 ; Smith et al, 1987 ; Aurousseau, 1986), de sorte que les acides gras issus de cette source ne peuvent guère contribuer à la masse adipeuse. En revanche, l'objectif recherché concernant la mise à disposition d'acides gras à nombre de carbones $<\mathrm{C} 15$ pour une utilisation énergétique pourrait interférer avec le métabolisme du glucose et celui des acides aminés (Richardson et al, 1979 ; Aurousseau et al, 1989), conduisant à un hyperinsulinisme comme le suggèrent les travaux de Crista et al (1979) et ceux de Waghorn et al (1987) concernant la liaison entre la disponibilité azotée et l'élévation de I'insulinémie. Dans ces conditions, l'ac- croissement de la proportion des matières grasses $(+4,4 \%)$, imposé dans la formulation, pourrait induire une situation d'insulino-résistance dans les cellules cibles par effet rétro-actif. Par ailleurs, les lipides saturés tendent à majorer l'insulino-résistance des adipocytes à court terme (Harris et Kor, 1992, chez le rat). Cette situation pourrait réduire la synthèse de novo et limiter l'activité des désaturases (Periago et al, 1989 ; Chang et al, 1992) lorsque le statut énergétique est favorable à la lipogenèse. En conséquence, l'accumulation des acides gras longs saturés d'origine alimentaire serait favorisée.

L'absence d'effet supplémentaire, enregistrée avec l'aliment supplémenté en saindoux, peut tenir à la faible différence dans la composition en acides gras des matières grasses présentes dans les aliments comparés. En effet, seule une modification des proportions des acides gras $\mathrm{C} 18=1$ et $\mathrm{C} 18=2$ apparaît entre Fs et Ft $(30,1 \%$ et $31,1 \%$, respectivement pour Fs vs $24,1 \%$ et $36,3 \%$ pour $\mathrm{Ft}$ ) ; les taux de matières grasses sont, de plus, assez proches (MG = $5 \%$ vs 3,7\% MS pour $\mathrm{Ft}$ ). Or, en raison de la biohydrogénation réalisée dans le rumen (Christie, 1981), ces valeurs semblent justifier d'un comportement identique.

\section{Couleur du tissu gras}

En ce qui concerne la coloration du tissu gras sous-cutané, aucune différence significative n'apparaît sur l'ensemble des profils de coloration quels que soient le type d'échantillonnage et le lot. Ponctuellement, cependant, le régime «coprah» $D_{(21)}$ tend à générer ultérieurement plus fréquemment des couleurs de type jaune clair que le régime témoin $(+15,5$ points de pourcentage $(P<0,08),+10,9$ points $(P<0,19)$ respectivement pour les échantillons 1 et 2). De même, le lot $D c_{(17)} F s$ paraît présenter plus souvent des carcasses bistre clair que les autres. Dans l'ensemble, la propor- 
tion de carcasses blanches est très faible $(<2 \%)$, en revanche celle de couleur à nuance jaune (bistre clair, jaune clair et bistre clair avec reflets verdâtres) est particulièrement élevée (entre 60 et $70 \%$ selon les lots). En conséquence, la tendance à l'amélioration de la couleur enregistrée dans les essais précédents, en présence d'huile de coprah ou de saindoux, n'est pas retrouvée. Une explication possible pourrait résider dans la procédure appliquée de ressuage accéléré. Cependant, les carcasses de «laitons» (agneaux non sevrés), traitées de la même façon, sont blanches (NCoul 1) pour la plupart. Ce constat pourrait être plutôt lié aux caractéristiques des matières premières utilisées pour fabriquer les aliments. Les proportions de luzerne déshydratée étaient notablement plus élevées dans les aliments complémentés en huile de coprah et en saindoux (respectivement $20 \%$ vs $10 \%$ pour $\mathrm{D}$ et $12 \%$ vs $10 \%$ pour F) ainsi que celles de pois dans l'aliment de finition Fs (15\% vs $10 \%)$. Les concentrations importantes de pigments caroténoïdes dans ces matières premières (Middenord et al, 1980), dont $75 \%$ sont sous forme de lutéine (Livingstone et al, 1969), pigment susceptible de colorer les graisses chez l'agneau (Prache et al, 1990), pourraient être à l'origine de ces fréquences élevées de couleur jaune clair et bistre clair dans le régime DcFs. En plus, l'accroissement du taux des matières grasses dans la ration et particulièrement des triglycérides riches en acides gras courts peut augmenter l'absorption de la lutéine (Hamilton, 1992). Or, l'acide laurique est en forte proportion dans l'aliment Dc $(27,0 \%$ de $M G$ vs $0,1 \%$ pour $\mathrm{Dt}$ ).

\section{REMERCIEMENTS}

Travail subventionné par le conseil régional de la région Midi-Pyrénées pour l'encouragement de la recherche appliquée ( $n^{\circ}$ de contrat 9000743).

\section{RÉFÉRENCES}

Andrews RP, Ørskov EE (1970) The nutrition of the early weaned lamb. II. The effect of dietary protein concentration, feeding level and sex on body composition at two live weights. J Agric Sci Camb 75, 19-26

Annison EF (1984) The metabolism of neutral acidic lipids by tissues of the ruminant. In : Herbivore Nutrition in the subtropics and tropics (FMC Gilchrist, RI Mackie, eds), Science Press, Craighall, Afrique du Sud, 549-570

Aurousseau B, Thériez M, Daniel M (1973) Influence de la nature des matières grasses incorporées dans l'aliment d'allaitement sur le métabolisme lipidique de l'agneau de bergerie. Ann Biol Anim Biochim Biophys 13, 93-105

Aurousseau B (1986) Influence des facteurs d'élevage sur l'état d'engraissement et la qualité des carcasses chez les Ovins. In : Qualités des produits chez les Ovins et les Caprins. 11e Journées rech ovine et caprine, INRA-ITOVIC, SPEOC, Paris, 210-235

Aurousseau $B$, Vermorel $M$, Thériez $M$, Vezinhet A (1989) Effects of substitution of tricaprylin or coconut oil for tallow in milk replacers offered to preruminant lambs. Ann Zootech 38, 49-59

Bauchart D, Doreau M, Legay-Carmier F (1985) Utilisation digestive des lipides et conséquences de leur introduction sur la digestion du ruminant. Bull Tech CRVZ Theix INRA 61, 65-77

Benevenga NJ, Steinman-Goldsworthy JK, Crenshaw TD, Odle J (1989) Utilization of medium-chain triglycerides by neonatal piglets. I. Effect on milk consumption and body fuel utilization. J Anim Sci 67, 3331-3339

Bénévent $M$ (1971) Croissance relative pondérale postnatale, dans les 2 sexes, des principaux tissus et organes de l'agneaux Mérinos d'Arles. Ann Biol Anim Bioch Biophys 11, 5-39

Bock BJ, Harmon DL, Brandt RT, Schneider Jr, Schneider JE (1991) Fat source and calcium level effects on finishing steer performance, digestion and metabolism. J Anim Sci 69, 2211-2224

Bouillier-Oudot M, Bozzolo G, Grasset D, Manse H (1992) Optimisation du jugement de la fermeté du gras de couverture des carcasses d'agneaux de bergerie. Ann Zootech 41, 187-203

Bozzolo G, Bouillier-Oudot M, Aoun M, Grasset D, Manse $H$ (1991) Incidence des acides gras alimentaires à chaîne moyenne, incorporés dans la régime de transition en post-sevrage, sur les caractéristiques de croissance et de carcasse d'agneaux sevrés précocement et engraissés intensivement en bergerie en été. Ann Zootech 40, 85-105

Bozzolo G, Bouillier-Oudot M, Quenardelle P, Grasset D, Manse $H$ (1992) Influence de l'incorporation de saindoux dans l'aliment concentré sur la croissance et les 
qualités de carcasse chez l'agneau mâle sevré précocement. Ann Zootech 41, 205-221

Bozzolo G, Bouillier-Oudot M, Candau M, El Hasbany G, Grasset D, Manse H (1993a) Effect of coconut oil in the post-weaning starter diet on growth and carcass qualities of male lambs, early weaned and intensively fattened in winter. Reprod Nutr Dev 33, 165181

Bozzolo G, Bouillier-Oudot M, Phrem G, Grasset D, Manse H (1993b) Effet de l'incorporation de saindoux dans le concentré d'engraissement sur la croissance et la qualité des carcasses chez l'agneau de bergerie. Cahiers Agricultures 2, 394-405

Cera KR, Mahan DC, Reinhart CA (1989) Apparent fat digestibilities and performance responses of postweaning swine fed diets supplemented with coconut oil, corn oil or tallow. J Anim Sci 67, 2040-2047

Chang JHP, Lunt DK, Smith SB (1992) Fatty acid composition and fatty acid elongase and stearoyl-CoA desaturase activities in tissues of steers fed high oleate sunflower seed. J Nutr 122, 2074-2080

Christie WW (1981) The effects of diet and other factors on the lipid composition of ruminant tissues and milk. In : Lipid Metabolism in Ruminant Animals (WW Christie, ed), Pergamon Press Publ, Oxford, Royaume-Uni, 193-225

Crista N, Gafton G, Garici I, Spatariu S (1979) Effect of abomasal added oils on glucose tolerance in lambs. Ann Rech Vet 10, 371-372

Devier CV, Pfander WH (1974) Source and level of dietary fat on fatty acid and cholesterol in lambs. J Anim Sci 38, 669-675

Foich J, Lees M, Sloane Stanley GH (1957) A simple method for the isolation and purification of total lipids from animal tissues. $J$ Biol Chem 226, 497-509

Frost SC, Wells MA (1981) A comparison of the utilization of medium and long chain fatty acids for oxidation and ketogenesis in the suckling rat: in vivo and in vitro studies. Arch Biochem Biophys 211, 357

Garton GA, Howell FDD, Duncan WRH (1972) Influence of dietary volatile fatty acid composition of lamb triglycerides with special reference to the effect of propionate on the presence of branched chain components. Br J Nutr 28, 409-416

Giger-Reverdin S, Aufrère J, Sauvant D, Demarquilly C, Vermorel M, Pochet S (1990) Prévision de la valeur énergétique des aliments composés pour les ruminants. INRA Prod Anim 3, 181-188

Hamilton PB (1992) The use of high-performance liquid chromatography for studying pigmentation. Poultry Sci $71,718-724$
Harris RBS, Kor H (1992) Insulin insensitivity is rapidly reversed in rats by reducing dietary fat from 40 to $30 \%$ of energy. J Nutr $122,1811-1822$

Livingstone AL, Kuzmicky DD, Knowles RE, Kohler GO (1969) The nature and deposition of the carotenoids from alfalfa and corn gluten meal in chicken skin. Poultry Sci 48, 1678-1683

Mahan DC (1991) Efficacy of initial postweaning diet and supplemental coconut oil or soybean oil for weanling swine. J Anim Sci 69, 1397-1402

Middendorf DF, Childs GR, Cravens WW (1980) Variation in biological availability of xanthophyll within and among genetic sources. Poulty Sci59, 1460-1470

Ørskov ER, Duncan WR, Carnie CA (1975) Cereal processing and food utilization by sheep. III. Effect of replacement whole barley by whole oats on food utilization and firmness and composition of subcutaneous fat. Anim Prod 21, 51-59

Periago JL, Sanchez del Castillo MA, Caamano G, Suarez $M$ (1989) Changes in lipid composition of liver microsomes and fatty acyl-CoA desaturase activities induced by medium chain triglyceride feeding. Lipids 24, 383-388

Prache S, Aurousseau B, Thériez M, Renerre M (1990) Les défauts de couleur du tissu adipeux sous-cutané des carcasses d'Ovins. INRA Prod Anim 3, 275-285

Richardson TC, Jeacock MK, Shepherd DAL (1979) Alanine and propionate metabolism in lamb liver. $A n n$ Rech Vét 10, 365-367

Smith SB, Jenkins T, Prior RL (1987) Carcass composition and adipose tissue metabolism in growing sheep. J Anim Sci 65, 1525-1530

St John LC, Lunt DK, Smith SB (1991) Fatty acid elongation and desaturation enzyme activites of bovine liver and subcutaneous adipose tissue microsomes. J Anim Sci 69, 1064-1073

Thériez M, Tissier M, Robelin J (1981) The chemical composition of the intensively fed lambs. Anim Prod $32,29-37$

Turgeon OA, Brink DR, Bartle SJ, Klopfenstein TJ, Ferrell CL (1986) Effects of growth rate and compensatory growth on body composition in lambs. J Anim Sci $63,770-780$

Waghorn GC, Flux DS, Ulyatt MJ (1987) Effect of dietary protein and energy intakes on growth hormone, insulin, glucose tolerance and fatty acid synthesis in young wether sheep. Anim Prod 44, 143-152

Wahle KWJ (1974) Desaturation of long-chain fatty acids by tissue preparations of the sheeps, rat and chicken. Comp Biochem Physiol 48B, 87-105 